\title{
USE OF POLLEN TETRADS FOR ROUTINE DNA DENSITOMETRY
}

\author{
A. KENTON \\ Cytology Section, Jodrell Laboratory, Royal Botanic Gardens, Kew, Richmond, Surrey, UK \\ Received 2.iii.84
}

\section{SUMMARY}

\begin{abstract}
Where meiosis results in balanced gametes, preparations of pollen tetrads stained with Feulgen provide a simple, consistent source for measuring $1 C$ DNA amount. The tetrad nuclear density is very close to that of root telophase nuclei, and the standard error of absorbance measurements is lower.

A technique for the preparation of tetrads for microdensitometry is described.
\end{abstract}

\section{INTRODUCTION}

DNA measurement by Feulgen densitometry is now a routine contribution to cytological investigations in plants. The most frequently used material has been root tips, when prophase $(4 C)$ or telophase $(2 C)$ nuclei have been measured. Some early results were published as DNA amount per cell (Baetke et al., 1967 and see Bennett and Smith, 1976); this estimate is, however, potentially unreliable, since values between $2 \mathrm{C}$ and $4 \mathrm{C}$ could be included (Bennett and Jellings, 1975). To minimise the risk of including values between 2C and $4 C$, Paroda and Rees (1971) measured presumed 2C nuclei from root tip meristems and then used the mean of the lowest 10 values.

Comparison of $4 \mathrm{C}$ values of prophases and metaphases of root tips has shown that metaphases give significantly lower values of absorption due to the increased density resulting from chromosomal contraction (Bennett and Jellings, 1975). However, there is no significant difference between $4 \mathrm{C}$ values measured at early and mid prophase, and it is this latter stage which is now routinely used in some laboratories (Bennett and Smith, 1976).

With the advent of more sophisticated equipment, it has become possible to measure DNA with greater accuracy, and most researchers now use relatively small numbers of $2 \mathrm{C}$ nuclei from several replicates (e.g., Seal and Rees, 1982; Narayan, 1982). Although root tip material is generally used, good results have also been obtained from leaf epidermal nuclei, whose $2 \mathrm{C}$ status has been established after comparison with root tip values (Price et al., 1970).

Tetrad nuclei from the second division of male meiosis would be expected to give a $1 \mathrm{C}$ value of DNA amount, since they represent the unreplicated haploid complement. This material does not generally appear to have been exploited for DNA measurement, although it may at times be more plentiful than root tip material and is easy to prepare, as the following study shows.

\section{Materials and Methods}

The material was taken from specimens in the living collection of the Jodrell Laboratory, Royal Botanic Gardens, Kew, and comprised Pelar- 
gonium "Picasso", Rhoeo spathacea "concolor" B23 and B24, Gibasis karwinskyana 10138 and $G$. pulchella 83.844 . The first four individuals are exclusively bivalent forming, the Rhoeo being a non-ring forming cytotype collected by Adams in Belize (Jones and Kenton, 1983). The Gibasis species were collected from the wild in Mexico by various Kew staff in 1979 and 1983. G. pulchella 83.844 regularly forms a quadrivalent at meiosis, which usually orientates in an alternate manner, and the plant has a pollen fertility of 70 per cent. Variation in nuclear mass attributable to irregular meiotic segregation is not therefore expected.

Tetrads were first spread onto slides and subsequently stained according to the method of Seal and Rees (1982). This allowed complete penetration of Feulgen, which was difficult to achieve when the anthers were stained intact.

Half an anther was removed with a needle from suitable buds and the pollen mother cells (PMC's) squeezed out into 1.8 per cent aceto-orcein and examined under a X10 objective. If tetrads were present, the remaining anthers were fixed in 4 per cent neutral formaldehyde for 2 hours, along with actively growing root tips from the same plant. After washing for 24 hours in two changes of distilled water, the material was transferred to $3: 1$ ethanol : acetic acid for $30 \mathrm{~min}$ to 24 hours. PMC's were then squeezed from the anthers onto a slide and a coverslip was placed in position. Gentle pressure and heat were applied to spread the tetrads slightly. The coverslips were removed with liquid $\mathrm{CO}_{2}$, and the slides dehydrated in ethanol and left to dry.

Both slides and root tips were washed in distilled water for $30 \mathrm{~min}$,

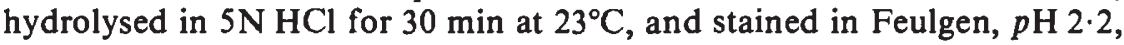
for 2 hours. After 3 washes of $10 \mathrm{~min}$ each in $\mathrm{SO}_{2}$ water, the slides were air dried and mounted in Euparal. Root tips were squashed in 45 per cent acetic acid and the coverslips removed as for anther material before mounting in Euparal. Readings of 50 tetrad nuclei and 50 root telophase nuclei from each plant were made immediately on a Vickers M85 scanning microdensitometer at $565 \mathrm{~nm}$.

\section{Results and Discussion}

Table 1 shows relative absorbance values for tetrad nuclei (presumed to be $1 C$ ) and root tip telophases (presumed 2C). Mean DNA amounts in picograms, estimated from root tip material in previous studies, have also been given for the species of Commelinaceae.

The $2 \mathrm{C}$ values are not, as expected, exactly double the tetrad values and in most cases, a $t$-test showed a significant difference between values for tetrads and half the value of root tip telophases. Fig. 1 shows histograms for tetrads and root telophase nuclei in each of the experimental species. In each case, the spread of values is greater for root tips than for tetrads, although the coefficient of variation, $s / \bar{x}$, may remain the same (table 1 ). Estimates for tetrads are usually (but not always) less than half the value of root tip telophases. There are several possible explanations for this:

(a) Differential contraction of nuclei within or between tissues could lead to an underestimate of absorbance in dense nuclei (cf. Verma and Rees, 1974); 


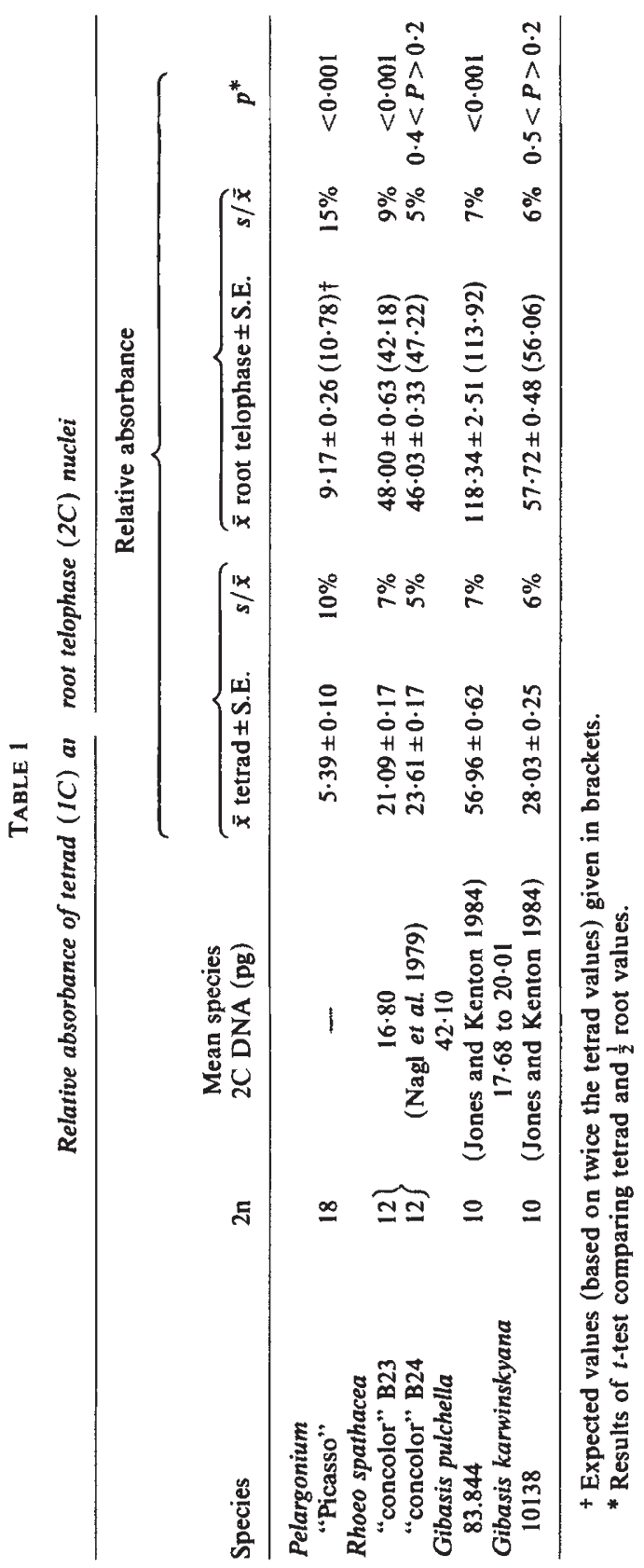



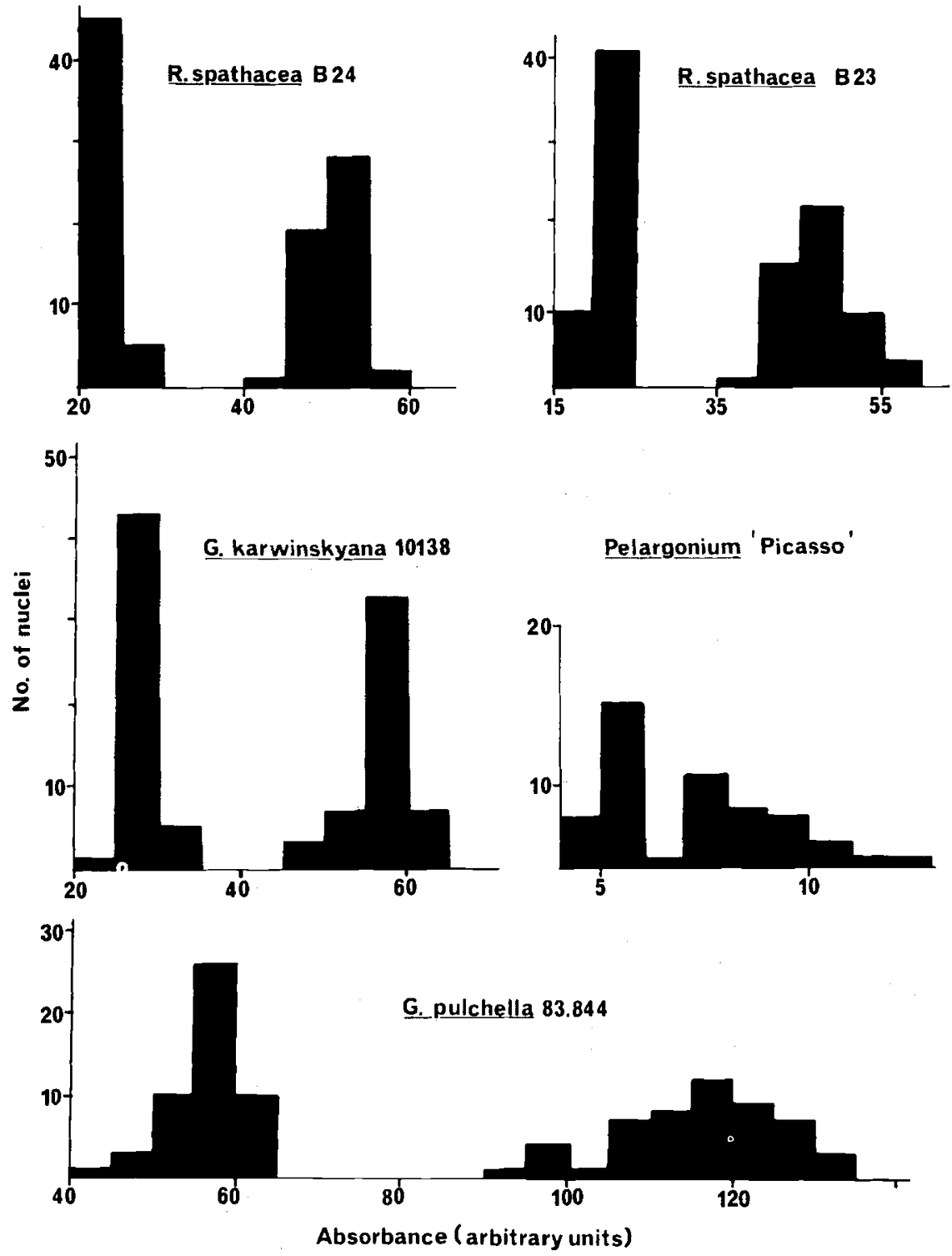

FIG. 1. Histograms showing the frequency of absorbance classes in tetrad nuclei (left) and root telophase nuclei (right) in the four experimental species.

(b) Nuclei in the centre of a clump of cells may not be critically focused relative to those at the edges;

(c) Replication may begin in the root tip nuclei before the daughter cells have separated, thus giving a greater degree of variability there. Any or all of these factors could also be responsible for the disparity between expected and observed values of absorbance in table 1 . 
In a comparison of recent and established allotetraploids of Brassica species, Verma and Rees (1974) demonstrated that in ancient allotetraploids, $2 \mathrm{C}$ values were underestimated due to an increase in nuclear density. This was realized by a positive and significant correlation between nuclear area and DNA amount measured by Feulgen densitometry. To test whether a change in density could be the cause of the disparity between tetrad and root tip nuclei, nuclear areas were plotted against Feulgen absorbance in two of the test species, Rhoeo spathacea B24 and Gibasis karwinskyana 10138 (figs. 2 and 3). The absorbance measurements were also plotted

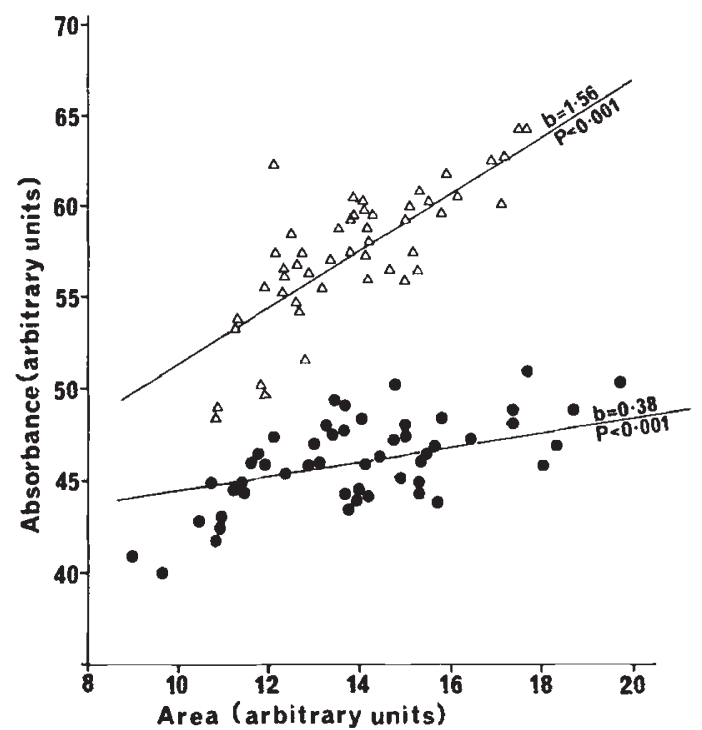

FIG. 2. Nuclear area plotted against Feulgen absorbance in root telophase nuclei of $G$. karwinskyana $10138(\triangle)$ and $R$. spathacea B24 (•).

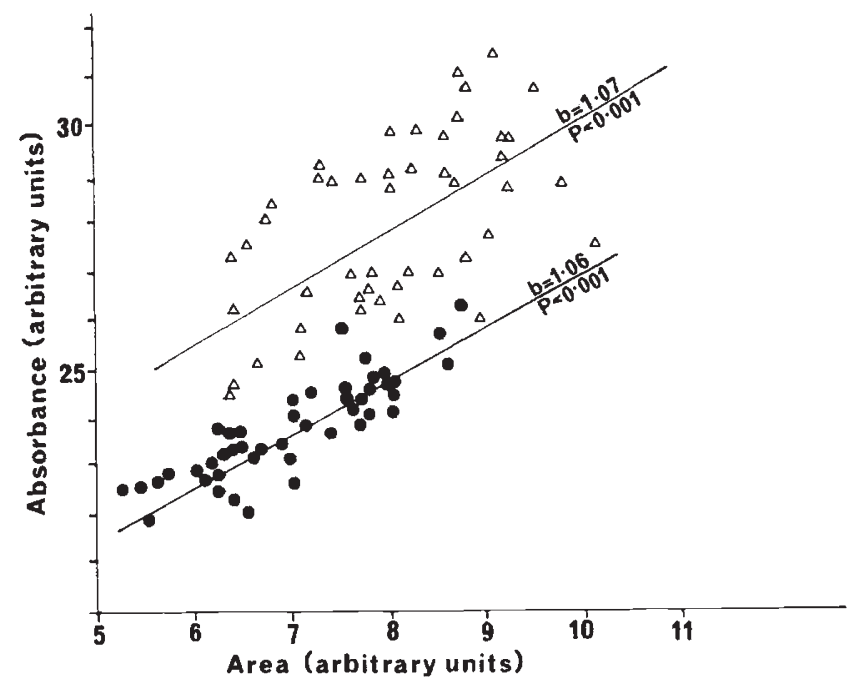

FIG. 3. Nuclear area plotted against Feulgen absorbance in tetrad nuclei of G. karwinskyana $10138(\triangle)$ and $R$. spathacea $B 24(\bullet)$. 


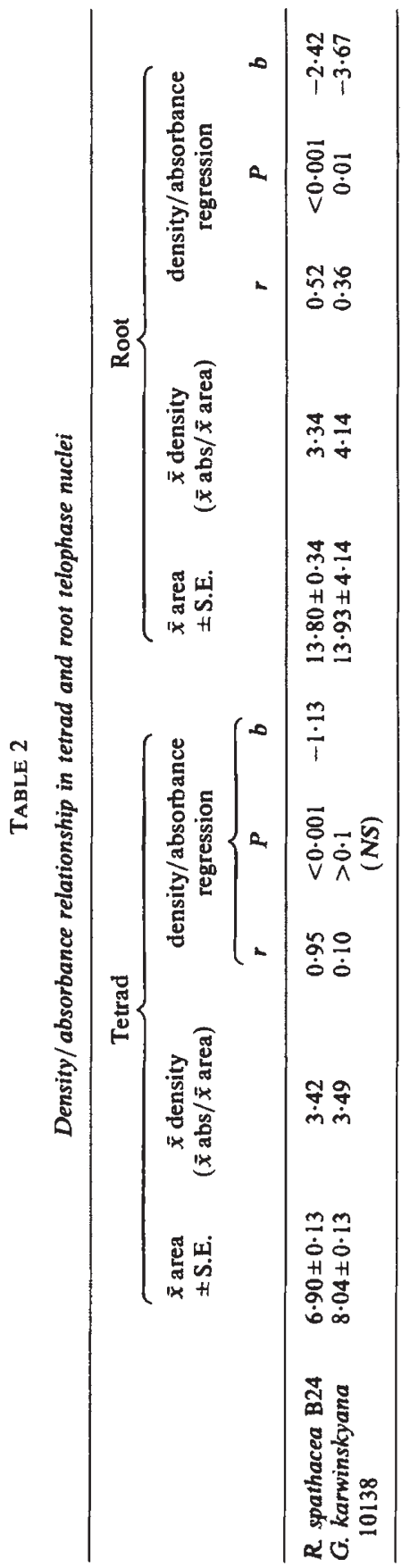


against nuclear density (measured as mean absorbance/mean are, table 2 and fig. 4), to see whether smaller nuclei did indeed give lower estimates of absorbance. The resulting regressions are summarised in table 2 and figs. 2-4. They show that in both species, there was a positive and significant correlation between absorbance and area (figs 2 and 3), and, in Rhoeo spathacea, a significant negative correlation between absorbance and density in both tetrad and root tip nuclei (fig. 4). The slopes of the regressions in

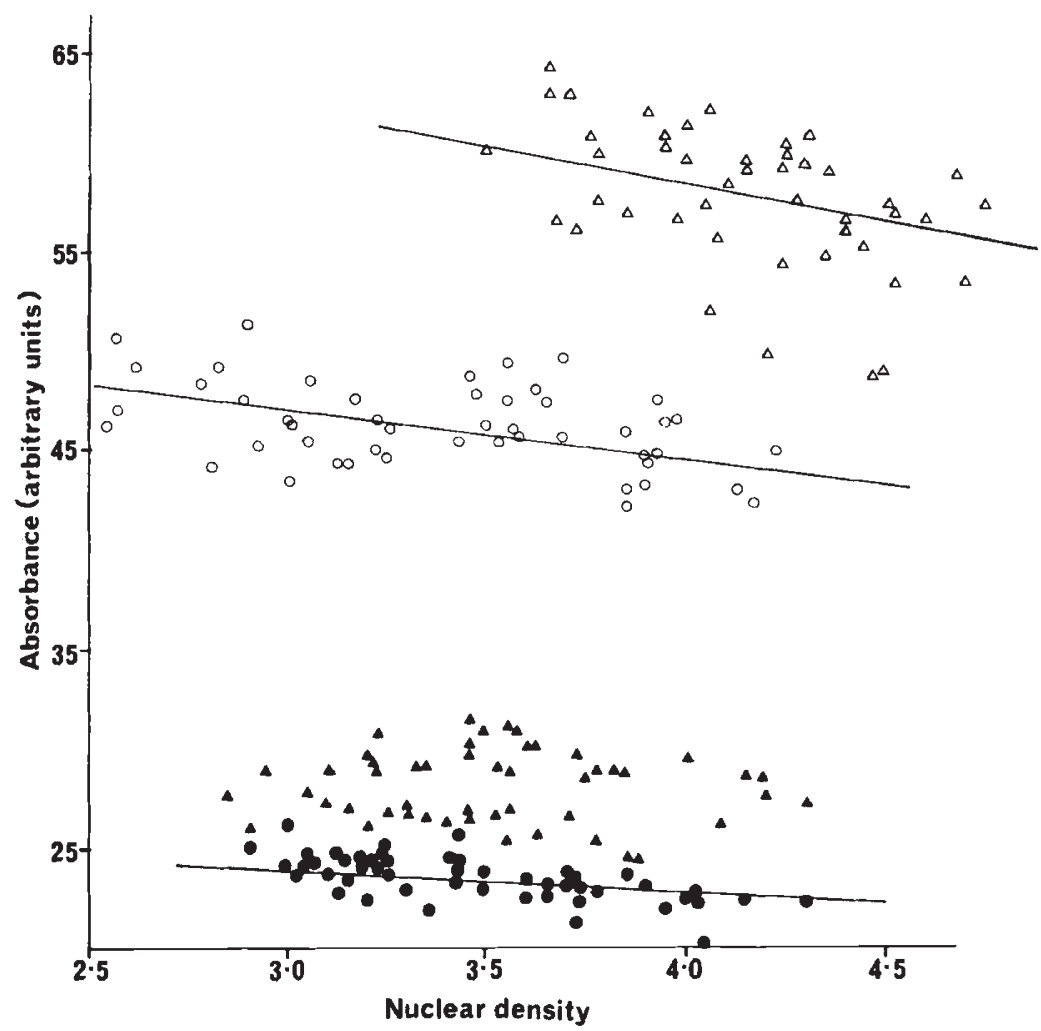

FIG. 4. Nuclear density (absorbance per unit area) plotted against Feulgen absorbance in tetrad and root telophase nuclei of $G$. karwinskyana 10138 and $R$. spathacea B24. $\Delta 10138$, tetrad; $\triangle 10138$, root tip; $\bigcirc$ B24, tetrad; B24, root tip. For details of the regressions, see table 2.

fig. 4 indicate that in the root tips of $R$ hoeo, there is a $2.4 \times$ decrease in absorbance with each unit of increase in density, while in tetrad nuclei, the decrease is 1.13 units for each unit of increase in density. In root tip nuclei the slope of the regression, $b$, is thus approximately twice that of the tetrads, as might be expected due to their double DNA amount. DNA amount does therefore tend to be underestimated in denser nuclei of both tetrads and root tips, at lest in Rhoeo. However, table 2 shows that the mean area of root tip nuclei in this species is exactly twice that of tetrad nuclei, and the mean density of the tetrads is very close to that of the root telophases. Underestimation of DNA amount due to a relatively greater density in tetrad nuclei would therefore appear to account neither for the discrepancy 
between observed and expected values of absorbance, nor for the greater amount of spread in root tip compared to tetrad values.

In G. karwinskyana, there was no significant correlation between density and absorbance in the tetrads (fig. 4, table 2 ), although a negative correlation $(\boldsymbol{P}=0.01)$ was shown in the root tips. Tetrad nuclei had a proportionally larger mean area, and their mean density was lower. If anything, therefore, the root tip nuclei would in this case appear to be denser than the tetrad nuclei. This again would not explain the low values of tetrad nuclei in terms of a relatively higher density.

In a root tip squash, the morphology of telophase nuclei varies considerably depending on the amount of pressure applied and the length of time elapsed since completion of the division. The experimenter thus has a greater degree of choice in selecting nuclei for measurement than in tetrad nuclei, where the morphology is rather uniform. Bennett (1976) noted that the rate of development of microspores was much slower than that of other meristematic tissue in the same species. Results to be published elsewhere also show that in the microspore development of $G$. pulchella, replication from $1 \mathrm{C}$ to $2 \mathrm{C}$ does not begin until the uninucleate pollen grains have been formed. The tetrad values may therefore be more stable for a longer period than the root tip telophases, which could offer an alternative explanation for the discrepancy between observed and expected values in table 1. A final factor worth consideration may be a statistical one; the lower variance in populations of tetrad nuceli (indicated by comparison of standard errors in table 1) may mean that an artificially high significance level is obtained when comparing them to the more variable root telophases.

DNA amounts in the tetrad nuclei were very uniform, even in the case of the quadrivalent-forming $G$. pulchella (fig. 1). In addition, the more effective spreading of the tetrads enabled clean preparations with very little background to be obtained. The fact that the nuclei were clustered in fours also allowed more rapid measurement. It is therefore suggested that when possible, tetrads would be a suitable material for routine measurement of plant DNA. However, it would be expedient to use a standard in which the density relationship between tetrad and root tip nuclei has been estimated.

Acknowledgements. I am grateful to Professor H. Rees and Professor K. Jones for their helpful suggestions and advice, and to Dr P. Brandham for critical appraisal of the manuscript.

\section{REFERENCES}

BAETKE, K. P., SPARROW, A. H. AND NAUMANN, C. H. 1967. The relationship of DNA content to nuclear and chromosome volumes and to radiosensitivity $\left(\mathrm{LD}_{50}\right)$. Proc. Nat. Acad. Sci. USA, 58, 533-540.

BENNETT, M. D. 1976. The cell in sporogenesis and spore development, In Yeoman, M. M. (ed.) Cell division in plants, Experimental Botany, 7, 161-198. Academic Press, London.

BENNETT, M. D. AND JELLINGS, A. J. 1975. DNA content of colchicine-induced endopolyploid nuclei in Vicia faba L. Heredity, 35, 261-272.

BENNETT, M. D. AND SMITH, J. B. 1976. Nuclear DNA amounts in angiosperms. Proc. $R$. Soc. Lond. B., 274, 227-274.

JONES, K. AND KENTON, A. 1983. Complex interchange heterozygotes and homozygotes in the Commelinaceae, In Brandham, P. E. and Bennett, M. D. (eds.) Kew Chromosome Conference II, George Allen and Unwin, London. 
JONES, K. AND KENTON, A. 1984. Mechanisms of chromosome change in the evolution of the tribe Tradescantieae (Commelinaceae). In Sharma, A. K. and Sharma A. (eds.) Chromosomes in Evolution of Eukaryotic Groups Vol. II, C.R.C. Press, Florida, pp. 143-168.

NAGL, W., FRISCH, B. AND FRÖLICH, E. 1979. Extra DNA during floral induction? Pl. Syst. Evol. Suppl. 2, 111-118.

NARAYAN, R. K. J. 1982. Discontinuous DNA variation in the evolution of plant species. Evolution, 36, 877-891.

PARODA, R. S. AND REES, H. 1971. Nuclear DNA variation in eu-sorghums. Chromosoma (Berl.), 32, 353-363.

PRICE, H. J., BACHMANN, K., CHAMBERS, K. L. AND RIGGS, J. 1980. Detection of intraspecific variation in nuclear DNA content in Microseris douglasii. Bot. Gaz., 141, 159-198.

SEAL, A. G. AND REES, H. 1982. The distribution of quantitative DNA changes associated with the evolution of diploid Festuceae. Heredity, 49, 179-190.

VERMA, S. C. AND REES, H. 1974. Nuclear DNA and the evolution of allotetraploid Brassicae. Heredity, 33, 61-68. 